\title{
Two thirds of the age-based changes in fluid and crystallized intelligence, perceptual speed, and memory in adulthood are shared
}

\author{
Paolo Ghisletta ${ }^{\mathrm{a}, \mathrm{b}, *}$, Patrick Rabbitt ${ }^{\mathrm{c}, \mathrm{d}}$, Mary Lunn ${ }^{\mathrm{e}}$, Ulman Lindenberger ${ }^{\mathrm{f}}$ \\ a Faculty of Psychology and Educational Sciences, University of Geneva, Boulevard du Pont d'Arve 40, 1211 Geneva, Switzerland \\ b Distance Learning University, Switzerland \\ c Department of Experimental Psychology, University of Oxford, 9 South Parks Road, Oxford, OX1 3UD, U.K. \\ d University of Western Australia, Perth, Australia \\ e Department of Statistics and St. Hughs' College, University of Oxford, 1 South Parks Road, Oxford, OX1 3TG, U.K. \\ ${ }^{\mathrm{f}}$ Center for Lifespan Development, Max Planck Institute for Human Development, Lentzeallee 94, 14195 Berlin, Germany
}

\section{A R T I C L E I N F O}

\section{Article history:}

Received 1 September 2010

Received in revised form 15 February 2012

Accepted 22 February 2012

Available online $\mathrm{xxxx}$

\section{Keywords:}

Cognition

Aging

Shared change

Longitudinal data

Multivariate multilevel model

\begin{abstract}
A B S T R A C T
Many aspects of cognition decline from middle to late adulthood, but the dimensionality and generality of this decline have rarely been examined. We analyzed 20-year longitudinal data of 6203 middle-aged to very old adults from Greater Manchester and Newcastle-upon-Tyne, UK. Participants were assessed up to eight times on 20 tasks of fluid intelligence, perceptual speed, memory, and vocabulary. We controlled for potential effects due to retest, city, sex, and socio-economic class. Average performance in all tasks declined with age, and individual differences in decline were present for all but one memory and two vocabulary tasks. Half of the variance in level of performance was shared across tasks. This proportion increased to $66 \%$ for individual differences in change. General level of performance and change therein correlated positively. We conclude that cognitive decline is heterogeneous across individuals and rather general at the within-individual level.
\end{abstract}

(c) 2012 Elsevier Inc. All rights reserved.
The dimensionality of cognitive change has long been the subject of cognitive aging research (Rabbitt, 1993). In particular, the question of the degree of generality in the rates of within-person changes within and across cognitive domains remains. The question "Is individual cognitive decline a general process, or is it differentially manifested across different domains?", albeit quite old, remains current.

The generality of adult cognitive decline can be investigated across and within persons. Across persons, the generality question can be phrased as, "Do all individuals eventually experience cognitive decline?" or "Is decline normative for the adult population?" Within persons, the question can be phrased as, "Does it all go together when it goes?" (Rabbitt, 1993 ) or "Does decline within a person tend to occur

\footnotetext{
* Corresponding author. Tel.: +4122 37991 31; fax: +4122 3799160. E-mail addresses: paolo.ghisletta@unige.ch (P. Ghisletta), patrick.rabbitt@psy.ox.ac.uk (P. Rabbitt), mary.lunn@st-hughs.ox.ac.uk (M. Lunn), lindenberger@mpib-berlin.mpg.de (U. Lindenberger).
}

simultaneously across different cognitive abilities?" The across-person question corresponds to the identification of interindividual differences in change (i.e., variance in change), and the within-person question corresponds to the analysis of interrelations in within-person change (covariances in change). Either question requires the analysis of longitudinal data to directly estimate within-person change, and betweenperson differences therein (Baltes \& Nesselroade, 1979).

Most recent analyses of longitudinal data indicate heterogeneity in rates of change and moderate to strong positive associations in such rates across different cognitive abilities (e.g., Anstey, Hofer, \& Luszcz, 2003; de Frias, Lövdén, Lindenberger, \& Nilsson, 2007; Ghisletta \& Lindenberger, 2003; Habib, Nyberg, \& Nilsson, 2007; Lindenberger \& Ghisletta, 2009; Tucker-Drob, 2011; Wilson et al., 2002). Individuals do not change uniformly; while some experience marked decline, others decline less, or may even stay stable (Habib et al., 2007). At the same time, the within-person patterns of change display some regularity across different 
cognitive domains. Typically, little to no intraindividual change is detected in crystallized abilities, while much within-person change in fluid abilities, speed, and memory exists. It is across this latter group of abilities that some associations in rates of change appear. For instance, Anstey et al. (2003) found relatively strong correlations among eightyear rates of change in speed and memory tasks $(r=.52)$ after statistically controlling for age, gender, education, depressive symptoms, general physical health, and medical condition. Wilson et al. (2002) analyzed six-year longitudinal behavioral data on episodic and semantic memory, working memory, perceptual speed, and visuospatial abilities tasks. They found that the rates of change in all domains correlated moderately to strongly, even when controlling for practice effects (from $r=.37$ to $r=.78$ ). Moreover, a principal component analyses revealed that the first component of the rates of change accounted for $61.8 \%$ of their variance. Lindenberger and Ghisletta (2009) found strong to very strong correlations across rates of change over 13 years in perceptual speed, memory, and verbal fluency tasks after control for age, time to death, and risk of dementia (from $r=.51$ to $r=1.00$, mean $r=.81$ ). Furthermore, an exploratory factor analysis revealed that $65 \%$ of the variance in these rates of change was accounted for by a common change factor. Finally, Tucker-Drob (2011) analyzed two-occasion data, assessed over up to seven years, on abstract reasoning, spatial visualization, episodic memory, and processing speed tasks, where each domain was assessed by three tasks. The author found that the latent change components of the four cognitive domains correlated moderately to strongly (from $r=.24$ to $r=.76$ ) and loaded substantively and positively on a common change factor. Moreover, the author concluded that change in the 12 cognitive tasks was on the average partially common (39\%), partially domain-specific (33\%), and partially task-specific (28\%). Also, it was found that the evidence for common change did not differ reliably between the young (18-49 years), middle aged (50-69 years), and older (7095 years) subsamples.

The reviewed recent longitudinal studies all applied the same type of statistical analysis, namely multilevel modeling (MLM) for longitudinal data (Laird \& Ware, 1982), which closely resembles the Latent Growth Model in the structural equation modeling literature (McArdle, 1986). The MLM contains two main components: the Intercept, which stands for general level of performance, and the Slope, representing change in performance. Intercept and Slope estimates describe both the average sample trajectory as well as the individuals' deviations from this trajectory.

The multivariate MLM, which is used here (see also Lindenberger \& Ghisletta, 2009) offers the additional advantage over multiple univariate MLMs of estimating the amount of interrelationships among the Intercepts and Slopes of all variables considered. In the present application, we are particularly interested in the correlations among all Slopes estimated in the multivariate MLM, which operationalize the associations in rates of change in cognitive performance.

The studies discussed above applied the MLM to study change as a function of occasions of measurement or participation time in the study. Age was simply considered as a focal covariate, which may modify the associations among rates of change. To study the dimensionality of cognitive aging, however, it is preferable to define change over chronological age (McArdle, 1988). This further augments the interpretability of the results in terms of developmental change, rather than mere passing of time, in cognitive performance during adulthood and advanced age, in accordance with lifespan theories of cognition (for a review see Lindenberger, 2001). Furthermore, given that the data are sparser when the time basis is chronological age rather than time in study or occasions of measurement, variances in slope often are not detected in the former situation. In other words, the statistical power to detect interindividual differences in intraindividual change is greater when change is described as a function of time in study or occasions of measurement than when change is described as a function chronological age. This also influences the power to detect slope covariances. Thus, inferences about associations among rates of change based on chronological age are more robust than when time in study or occasions of measurement are used as the time basis. This approach requires samples of aging individuals of considerable size that are measured over periods of time sufficiently long to exhibit change. An alternative and rather popular approach consists in estimating age effects on intraindividual change by comparing change gradients, defined over time in study or occasions of measurement, across different age groups.

Here, we analyze cognitive data from the University of Manchester Longitudinal Study (Rabbitt et al., 2004), a largescale 20-year longitudinal examination of cognitive performance in over 6000 healthy individuals initially aged 42 to 96 years. Some of the variables we considered have been analyzed in previous reports (e.g., Rabbitt, Lunn, Ibrahim, Cobain, \& McInnes, 2008a; Rabbitt, Lunn, Wong, \& Cobain, 2008b; Rabbitt, Lunn, Wong, \& Cobain, 2008c; Rabbitt et al., 2004). Those reports, however, did not focus on the multivariate relationships among the cognitive tasks, whereas those relationships are the focal interest in this paper. We investigate the amount of shared variance in cognitive change by applying the MLM statistical model to multiple markers of fluid and crystallized intelligence, perceptual speed, and memory. The large sample size, its broad coverage of age, and the breadth of the cognitive assessment allowed us studying cognitive change as a function of chronological age (instead of time of testing or occasions of measurement) over a range of different cognitive domains. We statistically controlled for various factors that often hamper the validity of age-based results in longitudinal studies, notably retest effects, and for variables potentially associated to cognitive performance (socio-economic status, sex, and place of residence).

\section{Method}

\subsection{Participants}

The analyses include a total of 6203 volunteering participants (4379 or $70.6 \%$ women), 2819 (45.4\%) of whom from Greater Manchester (UK) and 3384 from Newcastle-uponTyne (UK). The sample did not include individuals with severe visual or auditory handicaps. Participants with mild, correctable sensory handicaps were assessed with corrective devices. Socio-economic status was categorized according to the Registrar General's Scale of Occupational Categories (Office-of-Population-Censuses-and-Surveys, 1980) into six 
classes: professional (4.7\%), intermediate (31.6\%), non-manual skilled (26.8\%), manual skilled (21.6\%), partly skilled (7.4\%), and unskilled $(0.8 \%)$ - for the remaining $7.1 \%$ this information was missing or uncoded $(\mathrm{M} / \mathrm{U})$.

Age characteristics are shown at the end of Table 1 by occasions of measurement, with the actual sample sizes tested. There were two Test Batteries, A and B, each alternated biennially and administered at most four times. Both batteries were constructed to measure fluid, crystallized, visuo-spatial memory, and memory capacities with different tasks, so as to diminish retest effects (Rabbitt et al., 2004). About half the participants started with Test Battery A. Because we analyze change in cognitive performance as a function of chronological age the Test Battery order is trivial. From occasion 1 to 4 , the average age increased from 64.90 to 75.29 years, respectively, for Test Battery A, and from 67.69 to 77.10 years, respectively, for Test Battery B. Of the 6203 participants, 344 participated at each occasion of measurement, 574 provided data on 7 occasions, 362 on 6,578 on 5,802 on 4,787 on 3, 1011 on 2, and 1745 participants were assessed on only one occasion.

\subsection{Cognitive tasks}

Table 1 displays the names and abbreviations for all cognitive tasks. Here, we provide a short description of each cognitive task; for a detailed description, see Rabbitt (1993) and Rabbitt et al. (2004).

Test Battery A was composed of (a) two measures of general (fluid) intelligence, the Heim (1970) AH4-1 and 2; (b) two vocabulary tasks, the Raven (1965) Mill Hill Vocabulary A and B; and (c) three memory tasks, a verbal free recall, a cumulative verbal recall, and a picture recognition task.

Test Battery B included (a) a measure of general fluid intelligence, all four parts of the Cattell and Cattell (1960) Culture Fair Intelligence Test; (b) the WAIS-R vocabulary scale (Wechsler, 1986); (c) three speed tasks, a visual search task, the Savage (1984) Alphabet Coding Task, and the Semantic Reasoning task (Baddeley, Emslie, \& Nimmo-Smith, 1992); (d) four tasks of memory efficiency, from which five scores could be obtained, immediate verbal free recall, delayed verbal recall, semantic memory (a task called Propositions about People), and memory of objects and their positions; and (e) a task of visuo-spatial memory, from which three scores could be obtained, memory for shapes, for locations, and for both.

Table 1 also presents the standardized factor loadings of the 20 tasks when submitted to a confirmatory factor analysis in which fluid intelligence was measured by AH4-1, AH4-2, and CFT, crystallized intelligence by $\mathrm{MH}-\mathrm{A}, \mathrm{MH}-\mathrm{B}$, and WAISV, speed by VS, ACT, and SR, memory by VFR, CVR, PR, IVFR, DVR, PaP, MO, and MOP, and visuo-spatial memory by ShL, ShP, and ShSpL. This factorial representation obtained a satisfactory adjustment ( $\chi^{2}$ with 160 degrees of freedom of 3022, RMSEA $=.068, \mathrm{CFI}=.915, \mathrm{TLI}=.915, \mathrm{SRMR}=.052$ ). All tasks loaded positively and strongly on their factor except for PR. We also present the estimated reliability of each task based on the amount of variance accounted for by the underlying factor. Reliabilities are high for fluid and for crystallized tasks, moderate to high for visuo-spatial tasks, and low to moderate for memory tasks. The variables were quite symmetrically distributed (skewness between -1 and 1 ), except for visuo-spatial scores (skewness of -3.6 for ShL, -1.8 for $\mathrm{SpL}$, and -1.3 for $\mathrm{ShSpL}$ ). Test-retest reliabilities are given in Rabbitt et al. (2004) and are of similar orders of magnitude than the reliability estimates provided here.

\subsection{Testing procedures}

Both Test Batteries required two sessions (between one and four weeks apart) of about 90 min each for completion. The four waves of each battery were administered at about fouryear intervals. Participants were tested in groups of 5-20 individuals by two trained experimenters in well-lit, comfortable,

Table 1

Longitudinal design of the Manchester Study for cognitive variables.

\begin{tabular}{|c|c|c|c|c|c|c|c|c|c|}
\hline \multicolumn{5}{|c|}{ Test battery A } & \multicolumn{5}{|c|}{ Test battery B } \\
\hline Domain & $\lambda$ & $\mathrm{R}^{2}$ & Task & Abbreviation & Domain & $\lambda$ & $\mathrm{R}^{2}$ & Task & Abbreviation \\
\hline Gf & .91 & .83 & Heim Intelligence Test 1 & AH4-1 & Gf & .82 & .67 & Culture Fair Test & CFT \\
\hline Gf & .84 & .71 & Heim Intelligence Test 2 & AH4-2 & Gc & .81 & .65 & WAIS-R Vocabulary & WAISV \\
\hline Gc & .88 & .77 & Raven Mill Hill Voc. A & MH-A & Speed & .69 & .48 & Visual Search & VS \\
\hline Gc & .90 & .81 & Raven Mill Hill Voc. B & MH-B & Speed & .90 & .81 & Alphabet Coding Task & ACT \\
\hline Memory & .72 & .52 & Verbal Free Recall & VFR & Speed & .72 & .53 & Semantic Reasoning & SR \\
\hline Memory & .80 & .65 & Cumulative Verbal Recall & CVR & Memory & .74 & .55 & Imm. Verbal Free Recall & IVFR \\
\hline \multirow[t]{7}{*}{ Memory } & .41 & .17 & Picture Recognition & PR & Memory & .79 & .62 & Delayed Verbal Recall & DVR \\
\hline & & & & & VSMem & .69 & .48 & Shape Locations & ShL \\
\hline & & & & & VSMem & .91 & .82 & Spatial Locations & $\mathrm{SpL}$ \\
\hline & & & & & VSMem & .98 & .95 & Shape + Spatial Locations & ShSpL \\
\hline & & & & & Memory & .65 & .43 & Propositions about People & $\mathrm{PaP}$ \\
\hline & & & & & Memory & .67 & .44 & Memory Objects & MO \\
\hline & & & & & Memory & .64 & .41 & Memory Objects + Position & MOP \\
\hline
\end{tabular}

Measurement occasion

\begin{tabular}{llllllll}
\hline & 1 & 2 & 3 & 4 & 1 & 2 & 4 \\
\hline $\mathrm{N}$ & 5926 & 3771 & 2125 & 990 & 4258 & 2417 \\
Mean age (SD) & $64.90(7.45)$ & $68.53(6.87)$ & $72.23(6.41)$ & $75.29(5.91)$ & $67.69(7.05)$ & $72.50(6.38)$ & 7169 \\
{$[$ min-max] } & {$[43-93]$} & {$[49-92]$} & {$[54-93]$} & {$[62-97]$} & {$[42-96]$} & {$[47-95]$} & {$[51-96]$} \\
\hline
\end{tabular}

Note. $G f=$ fluid intelligence. $G c=$ crystallized intelligence. VSMem = visuo-spatial memory. Voc. $=$ Vocabulary. Imm. $=$ Immediate. $\lambda=$ standardized factor loading from confirmatory factor analysis. $\mathrm{R}^{2}=$ Task variance accounted for by domain-specific factor in confirmatory factor analysis. 
and quiet rooms. Only participants with sufficient visual and auditory functioning were retained. Participants could employ their usual corrective aids (e.g., glasses, hearing aids).

\subsection{Statistical analyses}

We applied a multivariate multilevel model (MMLM, MacCallum, Kim, Malarkey, \& Kiecolt-Glaser, 1997; McArdle, 1988), which defines for each cognitive task two components: the Intercept, representing the general level of performance, and the Slope, to represent change in performance. For both components the model estimates an overall sample average (fixed effects) and a sample variance (random effects), representing individual deviations around the sample average. The MMLM allows defining the associations between the random effects of the Intercepts and Slopes of all variables considered. These associations are expressed as covariances between each variable's random effects. All variables were standardized in T-metric ( mean $=50$, variance $=100$ ) with the time- 1 characteristics as reference.

The implementation of the MMLM can be quite problematic when the dimensionality of the data is high. Here, we have data on 6203 participants assessed up to 4 times on 20 variables. At most, we managed to estimate a MMLM with 12 variables. Fieuws and Verbeke (2006) showed how the likelihood of the full multivariate model, which cannot computationally be maximized, can be unbiasedly estimated by combining the estimates from all possible bivariate models. In a first step of analysis each possible bivariate multilevel model among a set of more than two dependent variables is estimated. Under maximum likelihood estimation, each bivariate model will estimate the parameter values that maximize the occurrence of the two dependent variables considered. Some parameters will be estimated only once (e.g., correlation between the Slope of the first and the second variable) and for such parameters hence no further step is required. Other parameters, however, will be estimated multiple times (e.g., mean of Slope of a variable). For these parameters a second step of analysis occurs, in which the estimates are combined to provide the most likely and unique estimated value. The authors have shown that parameters could be combined by averaging them to obtain the estimate with the lowest bias. Their simulations showed that this technique recovers the correct parameter estimates (for both the parameters estimated multiple times and those estimated only once). This method reduces hence drastically the dimensionality of the problem without loss in estimation quality. While very powerful, this method does however not eliminate all computational difficulties. In this application we hence tested all 190 possible bivariate MLMs (none of which engendered computational difficulties) plus the 20 univariate MLMs, for a total of 210 MLMs. We then combined their estimates to obtain the final results.

\subsubsection{Covariates in multivariate multilevel model}

The model included various covariates in order to (a) assure the appropriate use of Full Information Maximum Likelihood (FIML; Finkbeiner, 1979; also called raw maximum likelihood) estimation and (b) isolate aging effects from other influences that might lower internal validity. Demographic covariates included consideration of the participants' sex ( 0 for men, 1 for women), city (0 for Manchester, 1 for Newcastle-upon-Tyne), and socio-economic status (six dummy variables with the non-manual skilled occupation as the reference). Selectivity analyses revealed that participant's age, socio-economic status, previous cognitive scores and at times sex and city were highly related to dropout probability. Introducing such informative covariates in a regression model (hence, by extension also in a multilevel model) using FIML estimation greatly reduces the bias in parameter estimates, because all estimated effects are conditioned on the information that is associated to the reasons of dropout (Enders, 2001; Graham \& Donaldson, 1993). Another advantage of using FIML estimation is that it allows estimating both parameter values and their standard errors. Finally, FIML estimation is particularly well-suited in longitudinal analyses, where naturally previous measurements of the dependent variable of interest are included. Such previous values are almost always highly correlated with both the current value of the dependent variable and with the probability of dropout (Graham, 2009). Hence, attrition at later waves is clearly associated to previous values, and FIML estimation uses this information to obtain unbiased parameter estimates and standard errors (Graham, 2009).

For each cognitive variable we also included retest effects, not to underestimate aging effects. At occasions 2, 3, and 4 we included a dummy variable for each cognitive task to estimate the change in performance independent of aging effects. This technique is widely applied and has proved useful in a number of independent studies (e.g., Ferrer, Salthouse, McArdle, Stewart, \& Schwartz, 2005; Ghisletta \& de Ribaupierre, 2005; Lövdén, Ghisletta, \& Lindenberger, 2004; McArdle, Prescott, Hamagami, \& Horn, 1998; Rabbitt et al., 2004). However, this coding scheme does not allow estimating random effects in retest (else the model is overparameterized). We hence also tested a second coding scheme for retest effects, which assumed these effects to be linear (values 0,1,2,3 at occasions $1,2,3,4$, respectively) but variable (random) across participants. We compared the two retest effects and retained the more statistically adequate, if significant). We found random retest effects only for ACT and DVR. However, the age by retest effects interaction was not significant for DVR, while barely significant for ACT. In this latter case, however, the overall parameter estimates did virtually not change. We hence did not include age by retest effects interactions in the final model.

The focal covariates are of course those estimating aging effects. Chronological age was centered at 70 years, close to the overall average age. We also included quadratic effects by squaring centered chronological age. Random effects were specified for the Intercept and linear and quadratic aging effects, allowing cognitive performance to vary across participants with respect to their general level of performance, linear decline, and quadratic decline. Eq. (1) represents the MMLM, were $Y_{a i k}$ is the cognitive score at age $a$ for individual $i$ on the cognitive task $k$; $I, I S$, and $q S$ are the Intercept and the linear and quadratic Slopes, respectively; $\beta_{1,2,3}$ are three retest effects; $\beta_{4}$ is the city effect; $\beta_{5}$ estimates sex' effects; $\beta_{6,7,8,9,10,11}$ are the socio-economic class' effects; and $\varepsilon_{a i k}$ is the error component.

$$
\begin{aligned}
Y_{a i k}= & I_{i k}+I S_{i k} \cdot A_{a i}+q S_{k} \cdot A_{a i}^{2}+\beta_{(1,2,3) k} \cdot r_{(1,2,3) k}+\beta_{4 k} \cdot \operatorname{city}_{i} \\
& +\beta_{5 k} \cdot \operatorname{sex}_{i}+\beta_{(6,7,8,10,11) k} \cdot \operatorname{soc}_{(1,2,3,4,5,6) i k}+\varepsilon_{a i k}
\end{aligned}
$$


Eq. (2) expresses the same MMLM except that now there is a unique retest effect $\beta_{1 k i}$, which allows for individual differences

$$
\begin{aligned}
Y_{a i k}= & I_{i k}+l S_{i k} \cdot A_{a i}+q S_{k} \cdot A_{a i}^{2}+\beta_{1 k i} \cdot r_{k}+\beta_{2 k} \cdot c i t y_{i} \\
& +\beta_{3 k} \cdot \operatorname{sex}_{i}+\beta_{(4,5,6,7,8,9) k} \cdot \operatorname{soc}_{(1,2,3,4,5,6) i k}+\varepsilon_{a i k}
\end{aligned}
$$

The Intercept and the linear Slope (and the retest effect in Eq. (2)) have a subscript $i$, indicating that their magnitude was allowed to differ across individuals (i.e., individualspecific random effects). We did not find significant random effects for the quadratic Slope for any variable. We did not test interactions between the covariates because of no theoretical a-priori reason to do so and to avoid increasing further the models' complexity. The modeling procedure is the same as that used in previous reports of parts of these data (e.g., Rabbitt et al., 2004; 2008a; 2008b; 2008c).

We first analyzed each cognitive variable separately, to ascertain the best univariate MLM specifications. We found that for MH-B, WAISV, the two crystallized intelligence markers, and ShL there were no significant random effects for linear Slope. This informed the specification of the bivariate MLMs, in which each task's random effects of Intercept and, when present, linear Slope were allowed to covary. In the end, we obtained a $39 \times 39$ covariance matrix with 20 Intercepts, 17 Slopes, and 2 retest effects. This matrix was not positive definite. To reduce its dimensionality we hence could not use a confirmatory approach (such as confirmatory factor analysis), were limited to an exploratory analysis, and were not able to provide standard errors.

\subsubsection{Exploratory factor analysis}

We factor analyzed the $39 \times 39$ covariance matrix of random effects for Intercepts, Slopes, and retest effects to investigate the dimensionality of individual differences in aging effects on cognitive performance (cf. Lindenberger \& Ghisletta, 2009; Rowe \& Hill, 1998). The analyses on the $37 \times 37$ covariance matrix that excluded the two retest random effects produced virtually identical results with respect to the Intercept and Slope components.

\section{Results}

\subsection{Multivariate multilevel model}

Results (see Table 2) showed significant average (a) linear decline on all cognitive variables (ranging from -.60 for CFT to -.07 for MHA) and (b) quadratic decline on all tasks (from -.015 for PR to -.005 for MHB) except SR. Average retest effects were significant on all variables (from a low of .32 for CVR to a high of 7.97 for AH42). For all cognitive tasks but VS we found a difference in distribution of abilities between Newcastle-upon-Tyne and Manchester (from 3.86 for CFT to .43 for PR higher scores in Manchester); this effect may be due to sampling issues. There were significant sex differences in level in all tasks but MHB, ShL, and WAISV (ranging from a difference between women and men of -2.07 on SpL to 3.42 on CVR). The largest socio-economic differences were detected on MHA (ranging from -8.36 when in the lowest to 8.95 when in the highest class, compared to the average class).
Variability (random effects) was great for the Intercepts (from 36.87 for IVFR to 64.93 in ACT), while much smaller for linear age effects (from .020 for MHA to .181 for SpL; again, for MHB, WAISV, and ShL random effects of linear age were fixed at zero because of the results obtained in the preliminary univariate analyses). The two significant random effects for retest were of moderate size (2.72 for DVR and 3.23 for ACT).

In the end, the MMLM estimated all individuals' random effects among 20 Intercept and 17 linear Slope components and also two retest effects. To reduce the dimensionality of this $39 \times 39$ covariance matrix (with a total of 780 variances and covariances; cf. Supplementary Appendix) we performed an exploratory factor analysis.

\subsection{Exploratory factor analysis}

Table 3 presents the standardized factor loadings of the 20 Intercept, 17 linear Slope, and 2 retest random components when two correlated factors (with Promax rotation) were extracted. This solution accounted for $58 \%$ of the total reliable Intercept, linear Slope, and retest variance (61\% without the two retest effects). An alternative extraction based on the Kaiser-Guttman criterion resulted in 8 factors (see Table 4 for the eight eigenvalues of the EFA greater than one), which explained $36 \%$ of additional variance but had a less clear interpretation. The upper and lower panels show the standardized loadings of the Intercepts and Slopes, respectively, of the two-factor solution. For all cognitive tasks the loadings of the Intercept components on the general Intercept factor (factor 2 ) were very strong (mean $=.70$ ), while the loadings of the Intercept components on the general Slope factor (factor 1 ) were very weak (mean $=.02$, mean absolute value $=.11$ ). Likewise, across all cognitive tasks, the loadings of the linear Slope components on the general linear Slope factor were very strong (mean $=.79$ ), while those of the linear Slope components on the general Intercept factor were very weak (mean $=.04$, mean absolute value $=.15$ ).

The two factors correlated $.19(z=15.27, p<.001)$. The average squared multiple correlation of the Intercept components with respect to the general Intercept factor was .50, while the analogous measure for the linear Slope components to the general linear Slope factor was .66. Thus, in this study, nearly two thirds of the amount of reliable age-based change in fluid and crystallized intelligence, perceptual speed, and memory in adulthood are shared.

\section{Discussion}

This study examined cognitive decline in a relatively wide spectrum of abilities (memory, perceptual speed, fluid intelligence, crystallized intelligence, and vocabulary) with standardized tasks administered in a within-person design to a large sample of mid- to very old adults up to four times each, over an age period of up to 18 years. The statistical model adopted, the MMLM, allowed us estimating the degree of association between general level and rates of linear change on the cognitive variables marking different domains of cognitive performance, controlling for retest, city, sex, and socioeconomic class' effects. 
Application of the MMLM helped to replicate a number of extant results (e.g., Anstey et al., 2003; Habib et al., 2007; Lindenberger \& Ghisletta, 2009; Rabbitt, 1993; Tucker-Drob, 2011; Wilson et al., 2002). First, we found age-related decline in all variables, including the two vocabulary tasks (the typical markers of age-resistant crystallized intelligence). Second, we found that individuals differ in their rate of cognitive decline. Some of the factors promoting individual differences in rates of cognitive decline may relate to lifestyle (e.g. Hertzog, Kramer, Wilson, \& Lindenberger, 2008), cognitive resources (Bäckman, Small, Wahlin, \& Larsson, 2000), diseases (e.g, Rabbitt et al., 2008a), impending death (e.g., Ghisletta, McArdle, \& Lindenberger, 2006; Rabbitt et al., 2008c), common genetic variation (e.g., Lindenberger et al., 2008), or senescent changes of the brain (e.g., Raz et al., 2005). Lindenberger et al. (2008) recently put forward the hypothesis that neurochemical and neuroanatomical brain resources may modulate the influence of common genetic polymorphisms on cognitive performance in a nonlinear fashion.
This influence appears weak during young adulthood, when cognitive performance is at its peak, and in very old age, when terminal decline and dementia become prevalent. The strongest influence of common genetic polymorphisms on cognitive functioning would appear somewhere in between, during mid and old age. In agreement with this hypothesis, Li et al. (2010) found that the common Val66Met missense polymorphism of the brain-derived neurotrophic factor gene (BDNF) gene reliably affects individual differences in backward serial recall in older adults, but not in younger adults. While the present analyses cannot differentiate among these and additional influences on differential cognitive change, they strongly suggest that different individuals are affected by such influences to different degrees.

The major result of the present analyses is that nearly two thirds of the variance in individual differences in cognitive decline were shared across all variables evincing differential individual decline. This finding speaks to the second question of the study, "Does decline within a person tend to occur

Table 2

Parameter estimates (and standard errors) of the multivariate multilevel model.

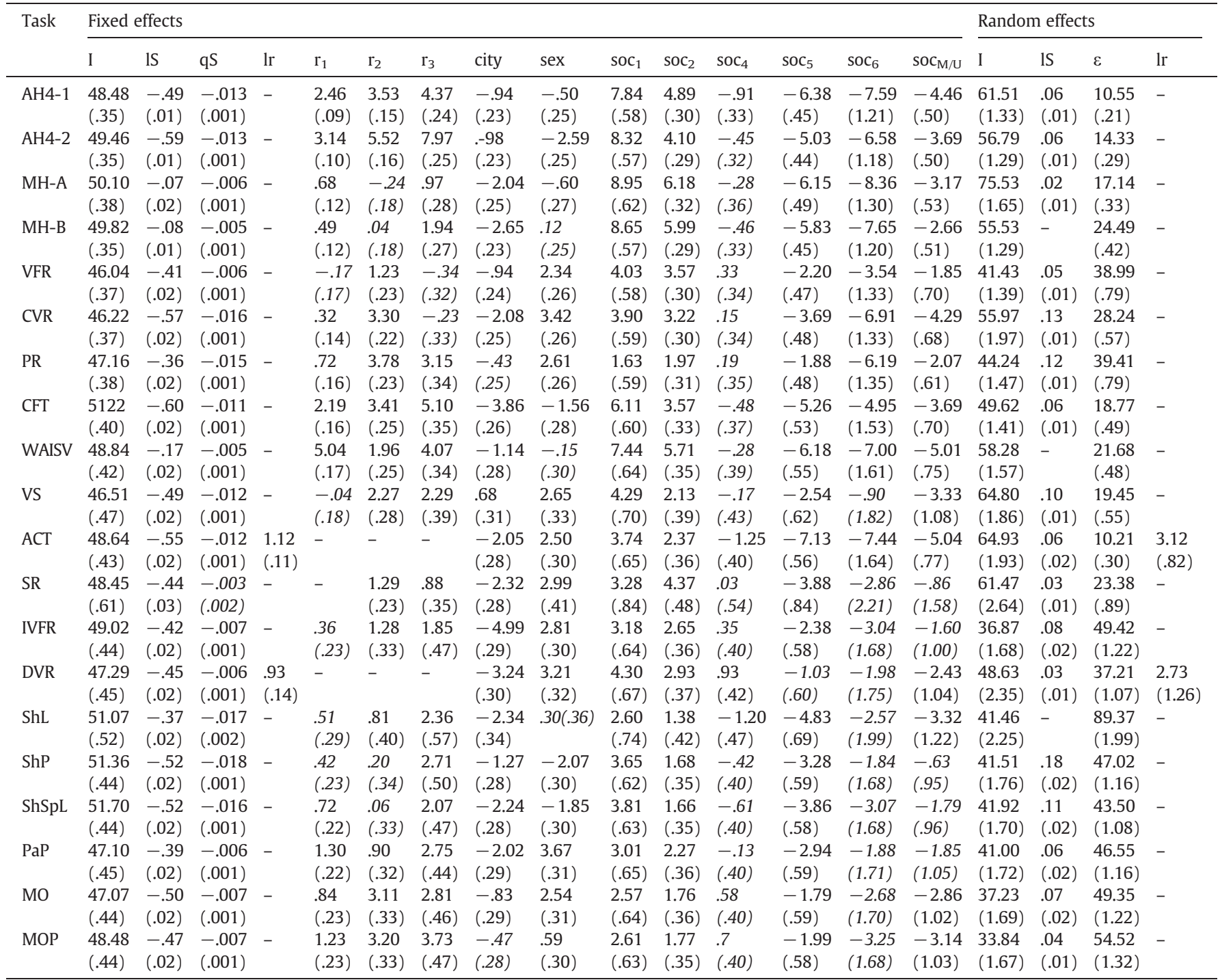

Note. Random effects are variances. $\mathrm{I}=$ Intercept. $\mathrm{IS}=$ linear Slope. $\mathrm{q} S=$ quadratic Slope. $\mathrm{lr}=$ linear retest effect. $\mathrm{r}_{1}=$ first retest effect. $\mathrm{r}_{2}=$ second retest effect.

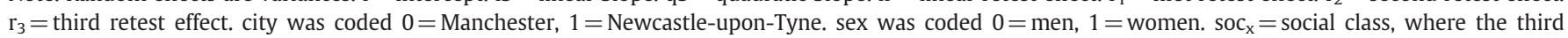
occupational category was used as comparison. $\operatorname{soc}_{\mathrm{M} / \mathrm{U}}=$ social class information missing or uncoded. $\varepsilon=$ Error component. Non-significant effects are in italic. 
Table 3

Factor loadings of the two-factor exploratory analysis on the covariance matrix of variables' intercepts and slopes (with Promax rotation; the two factors correlate .19).

\begin{tabular}{|c|c|c|c|}
\hline & & Factor1 & Factor2 \\
\hline \multirow[t]{20}{*}{ Intercepts } & AH4-1 & -0.12 & 0.88 \\
\hline & AH4-2 & 0.01 & 0.77 \\
\hline & MH-A & -0.15 & 0.72 \\
\hline & MH-B & -0.19 & 0.75 \\
\hline & VFR & 0.02 & 0.69 \\
\hline & CVR & 0.18 & 0.75 \\
\hline & PR & 0.04 & 0.45 \\
\hline & CFT & -0.05 & 0.83 \\
\hline & WAISV & -0.17 & 0.80 \\
\hline & VS & 0.10 & 0.50 \\
\hline & ACT & 0.03 & 0.77 \\
\hline & SR & 0.11 & 0.61 \\
\hline & IVFR & 0.11 & 0.68 \\
\hline & DVR & 0.03 & 0.68 \\
\hline & ShL & -0.20 & 0.69 \\
\hline & $\mathrm{SpL}$ & 0.20 & 0.70 \\
\hline & ShSpL & 0.16 & 0.72 \\
\hline & $\mathrm{PaP}$ & 0.02 & 0.66 \\
\hline & MO & 0.17 & 0.64 \\
\hline & MOP & 0.14 & 0.69 \\
\hline \multirow[t]{17}{*}{ Slopes } & AH4-1 & 0.75 & 0.09 \\
\hline & AH4-2 & 0.90 & 0.01 \\
\hline & MH-A & 0.51 & 0.41 \\
\hline & VFR & 0.84 & -0.36 \\
\hline & CVR & 1.02 & 0.00 \\
\hline & PR & 0.79 & -0.06 \\
\hline & CFT & 0.84 & 0.23 \\
\hline & VS & 0.68 & -0.06 \\
\hline & ACT & 0.84 & 0.06 \\
\hline & SR & 0.73 & 0.05 \\
\hline & IVFR & 1.03 & 0.10 \\
\hline & DVR & 1.10 & -0.09 \\
\hline & $\mathrm{SpL}$ & 0.38 & 0.31 \\
\hline & ShSpL & 0.47 & 0.28 \\
\hline & $\mathrm{PaP}$ & 0.88 & -0.21 \\
\hline & MO & 0.84 & 0.09 \\
\hline & MOP & 0.85 & -0.09 \\
\hline \multirow[t]{2}{*}{ Linear retest } & ACT & -0.04 & 0.06 \\
\hline & DVR & 0.24 & 0.13 \\
\hline
\end{tabular}

Note. First block denotes Intercepts, the second Slopes, the third linear retest effects (only present for ACT and DVR).

simultaneously across different cognitive abilities?" By and large, to the extent that the variables analyzed here represent fluid and crystallized intelligence, perceptual speed, and memory (cf. Table 1 ), the answer to this question tends to be positive. The commonality in the rates of age-related decline may reflect normative senescent changes in neurochemical, anatomical, and functional brain resources that drive cognitive decline in the domains of fluid intelligence, perceptual speed, and memory. Some of the relevant mechanisms may include neuronal cell loss, dendritic degeneration, synapse reduction, and reduction in dopaminergic neurotransmission.

Note, however, that the present results leave considerable room for domain-specific mechanisms, accounting for the remaining $34 \%$ of non-shared variance in change (cf. Anstey et al., 2003 and Tucker-Drob, 2011). That is, we do not want to promote the perspective that all variance in change is shared across a wide variety of cognitive domains. Put differently, decline on a given cognitive task does not inevitably imply decline on all other tasks. Whatever mechanisms that drive normative senescent changes, they are not exclusively
Table 4

Eight eigenvalues greater than one of the exploratory factor analysis on the covariance matrix of variables' Intercepts, Slopes, and retest components.

\begin{tabular}{lll}
\hline Eigenvalue & Proportion & Cumulative \\
\hline 14.25 & 0.37 & 0.37 \\
8.92 & 0.23 & 0.59 \\
3.83 & 0.10 & 0.69 \\
2.61 & 0.07 & 0.76 \\
2.46 & 0.06 & 0.82 \\
2.16 & 0.06 & 0.88 \\
1.53 & 0.04 & 0.92 \\
1.29 & 0.03 & 0.95 \\
\hline
\end{tabular}

general. This perspective is coherent with recent results by Tucker-Drob (2011), who found that while two fifths of change in cognitive performance appears global, one third is domain-specific and about three tenths are task-specific.

\subsection{Limitations}

The final results of this report stem from an exploratory factor analysis of a moment matrix that was not positive definite. This limits the strength of our conclusions, in that we could not offer inferential results (standard errors, $p$-values) about our parameter estimates and could not statistically compare alternative hypotheses. This situation is however typical in large multivariate design (20 variables) assessed longitudinally (up to four times each) over long intervals of time (up to 20 years). Current statistical software and implemented optimization techniques do not allow analyzing such a large data set in a confirmatory fashion. Trying to do so would inevitably involve such a large set of parameter restrictions that in the end the results would be biased and lose validity. Alternatively, we could have chosen a subset of variables to analyze only so as to obtain a well-behaved solution. In that case generalizability of the study would have been dramatically reduced.

Our analyses relied on a number of assumptions. First, because we used FIML estimation we assumed that missing data were not associated to variables other than those included in the analyses. This assumption is quite typical in cognitive aging research, given that prior cognitive performance, age, and the various covariates in the model (socio-economic status, sex and city of residence) are highly informative of study participation. We furthermore assumed that the sample forms a homogeneous group, other than for the covariates considered. That is, we assumed that the major sources of explained heterogeneity in our sample were either directly measured by or highly related to the variables included in the analyses and not on other, external variables. Both assumptions would have been less restrictive if additional relevant covariates had been added to the analysis (e.g., medical history, health, biological and genetic information). In other words, participants could differ with respect to variables other than those we included in the multivariate model, and such differences could be reflected on different estimates of the model's parameters. We also considered potential retest effects that were limited to the variables analyzed here. Some participants undertook cognitive tasks other than those analyzed here, and potentially these may have produced positive transfer effects. Such transfer effects 
are, however, generally quite weak, and, if present, usually originate from specifically trained skills, absent in the tasks analyzed here (Hertzog et al., 2008). Finally, while the initial longitudinal excluded individuals with dementia, some developed the disease during the period of observation. However, up to 2008 only nine participants among the 2819 from Manchester were reported having dementia (for five individuals the death certificate cited dementia, while an additional four were screened positive for dementia at a regular semiyearly screening; Rabbitt et al., 2008b). We lack the exact number for the Newcastle participants, but if they present a similar prevalence, there should be 11 cases, for a total of 20 demented individuals in the overall sample. This number is likely to be an underestimation, as death certificates may not systematically cite the presence of this disease. We nevertheless think that the main conclusions of this study are not invalidated by unaccounted dementia cases.

\subsection{Conclusions}

The present analyses are the most comprehensive of the University of Manchester Longitudinal Study (Rabbitt et al., 2004). Several previous reports of parts of the data analyzed here are available (e.g., Rabbitt et al., 2004, 2008a, 2008b, 2008c) and each has focused on the cognitive tasks individually. Some aspects of the analyses presented here have been discussed with respect to a subset of the cognitive tasks in such reports. The unique contribution of these analyses was, for the first time, to analyze (a) all 20 cognitive variables together and (b) not separately but concomitantly, to assess their interrelationships in time. This represents, to our knowledge, the largest analyses of cognitive aging data, encompassing 20 cognitive tasks, stemming from at least four large cognitive domains, assessed over a 20-year period on more than 6000 participants. While we presented strong evidence that the dimensionality of cognitive aging is low, we cannot, based on the present behavioral evidence, draw strong conclusions about the number and nature of the underlying driving factors. Future interdisciplinary research projects need to investigate cognitive decline assessed with well-known standardized behavioral measures in relation to neuroanatomical and neurochemical changes, genetic makeup, medical information, and lifestyle factors. In parallel, technical advances in the implementation of statistical models to very large and often challenging data sets are needed. Such rich interdisciplinary projects and technical progress ought to provide promising new evidence to advance scientific knowledge about cognitive aging.

Supplementary materials related to this article can be found online at doi:10.1016/j.intell.2012.02.008.

\section{Acknowledgments}

We would like to thank Prof. Geert Verbeeke for the precious advice on the estimation of the final multivariate multilevel model.

\section{References}

Anstey, K. J., Hofer, S. M., \& Luszcz, M. A. (2003). A latent growth curve analysis of late-life sensory and cognitive function over 8 years: Evidence from specific and common factors underlying change. Psychology and Aging, 18, 714-726.
Bäckman, L., Small, B. J., Wahlin, A., \& Larsson, M. (2000). Cognitive functioning in very old age. In F. I. M. Craik, \& T. A. Salthouse (Eds.), The handbook of aging and cognition (pp. 499-558). (2nd ed.). Mahwah, N.J.: Lawrence Erlbaum Associates.

Baddeley, A. D., Emslie, H., \& Nimmo-Smith, I. (1992). The Speech and Capacity of Language Processing (SCOLP) test. Bury St. Edmunds, UK: Thames Valley Test Company.

Baltes, P. B., \& Nesselroade, J. R. (1979). History and rationale of longitudinal research. In J. R. Nesselroade \& P.B. Baltes (Eds.), Longitudinal research in the study of behavior and development (pp. 1-39). New York, NY: Academic Press.

Cattell, R. B., \& Cattell, A. K. S. (1960). The Individual or Group Culture Fair Intelligence Test. Champaign, IL.: IPAT.

de Frias, C. M., Lövdén, M., Lindenberger, U., \& Nilsson, L. G. (2007). Revisiting the dedifferentiation hypothesis with longitudinal multi-cohort data. Intelligence, 35, 381-392.

Enders, C. K. (2001). A primer on maximum likelihood algorithms available for use with missing data. Structural Equation Modeling, 8, 128-141.

Ferrer, E., Salthouse, T. A., McArdle, J. J., Stewart, W. F., \& Schwartz, B. S. (2005). Multivariate modeling of age and retest in longitudinal studies of cognitive abilities. Psychology and Aging, 20, 412-422.

Fieuws, S., \& Verbeke, G. (2006). Pairwise fitting of mixed models for the joint modeling of multivariate longitudinal profiles. Biometrics, 62(2), 424-431.

Finkbeiner, C. (1979). Estimation for the multiple factor model when data are missing. Psychometrika, 44, 409-420.

Ghisletta, P., \& de Ribaupierre, A. (2005). A dynamic investigation of cognitive dedifferentiation with control for retest: Evidence from the Swiss Interdisciplinary Longitudinal Study on the Oldest Old. Psychology and Aging, 20, 671-682.

Ghisletta, P., \& Lindenberger, U. (2003). Age-based structural dynamics between perceptual speed and knowledge in the Berlin Aging Study: Direct evidence for ability dedifferentiation in old age. Psychology and Aging, 18, 696-713.

Ghisletta, P., McArdle, J. J., \& Lindenberger, U. (2006). Longitudinal cognition-survival relations in old and very old age: 13 -year data from the Berlin Aging Study. European Psychologist, 11, 204-223.

Graham, J. W. (2009). Missing data analysis: Making it work in the real world. Annual Review of Psychology, 60, 549-576.

Graham, J. W., \& Donaldson, S. I. (1993). Evaluating interventions with differential attrition: the importance of nonresponse mechanisms and use of follow-up data. Journal of Applied Psychology, 78, 119-128.

Habib, R., Nyberg, L., \& Nilsson, L. -G. (2007). Cognitive and non-cognitive factors contributing to the longitudinal identification of successful older adults in the Betula study. Aging Neuropsychology and Cognition, 14, 257-273.

Heim, A. W. (1970). AH4 group test of general intelligence. Windsor, UK.: NFER-Nelson.

Hertzog, C., Kramer, A. F., Wilson, R. S., \& Lindenberger, U. (2008). Enrichment effects on adult cognitive development: Can the functional capacity of older adults be preserved and enhanced? Psychological Science in the Public Interest, 9, 1-65.

Laird, N. M., \& Ware, J. H. (1982). Random-effects models for longitudinal data. Biometrics, 38, 963-974.

Li, S. -C., Chicherio, C., Nyberg, L., von Oertzen, T., Nagel, I. E., Papenberg, G., Sander, T., et al. (2010). Ebbinghaus revisited: influences of the BDNF Val66Met polymorphism on backward serial recall are modulated by human aging. Journal of Cognitive Neuroscience, 22, 2164-2173.

Lindenberger, U. (2001). Lifespan theories of cognitive development. In N. J. Smelser, \& P. B. Baltes (Eds.), International encyclopedia of the social and behavioral sciences (pp. 8848-8854). Oxford, UK: Elsevier.

Lindenberger, U., \& Ghisletta, P. (2009). Cognitive and sensory declines in old age: Gauging the evidence for a common cause. Psychology and Aging, 24, 1-16.

Lindenberger, U., Nagel, I. E., Chicherio, C., Li, S. -C., Heekeren, H. R., \& Bäckman, L. (2008). Age-related functioning in brain resources modulates genetic effects on cognitive functioning. Frontiers in Neuroscience, 2, 234-244.

Lövdén, M., Ghisletta, P., \& Lindenberger, U. (2004). Cognition in the Berlin Aging Study (BASE): The first ten years. Aging Neuropsychology and Cognition, 11, 104-133.

MacCallum, R. C., Kim, C., Malarkey, W. B., \& Kiecolt-Glaser, J. K. (1997). Studying multivariate change using multilevel models and latent curve models. Multivariate Behavioral Research, 32, 215-253.

McArdle, J. J. (1986). Latent growth within behavior genetic models. Behavior Genetics, 16, 163-200.

McArdle, J. J. (1988). Dynamic but structural equation modeling of repeated measures data. In J. R. Nesselroade, \& R. B. Cattell (Eds.), Handbook of multivariate experimental psychology (pp. 561-614). New York: Plenum Press. 
McArdle, J. J., Prescott, C. A., Hamagami, F., \& Horn, J. L. (1998). A contemporary method for developmental-genetic analyses of age changes in intellectual abilities. Developmental Neuropsychology, 14, 69-114.

Office-of-Population-Censuses-and-Surveys (1980). Classification of occupations 1980. London, U.K.: Her Majesty's Stationery Office.

Rabbitt, P. M. A. (1993). Does it all go together when it goes? The nineteenth Bartlett Memorial Lecture. The Quarterly Journal of Experimental Psychology. A, 46, 385-434.

Rabbitt, P. M. A., Lunn, D., Ibrahim, S., Cobain, M., \& McInnes, L. (2008). Unhappiness, health and cognitive ability in old age. Psychological Medicine, 38, 229-236.

Rabbitt, P. M. A., Lunn, M., Wong, D., \& Cobain, M. (2008). Sudden declines in intelligence in old age predict death and dropout from longitudinal studies. Journal of Gerontology Psychological Sciences, 63B, P205-P211.

Rabbitt, P. M. A., Lunn, M., Wong, D., \& Cobain, M. (2008). Age and ability affect practice gains in longitudinal studies of cognitive change. Journal of Gerontology Psychological Sciences, 63B, P235-P240.

Rabbitt, P. M. A., McInnes, L., Diggle, P., Holland, F., Bent, N., Abson, V., et al. (2004). The University of Manchester Longitudinal Study of Cognition and Normal Health Old Age, 1983 through 2003. Aging Neuropsychology and Cognition, 11, 245-279.

Raven, J. C. (1965). The Mill Hill Vocabulary scale. London, U.K.: H. K. Lewis.

Raz, N., Lindenberger, U., Rodrigue, K. M., Kennedy, K. M., Head, D. Williamson, A., et al. (2005). Regional brain changes in aging healthy adults: General trends, individual differences, and modifiers. Cerebral Cortex, 15, 1676-1689.

Rowe, K. J., \& Hill, P. W. (1998). Modeling educational effectiveness in classrooms: The use of multi-level structural equations to model students' progress. Educational Research and Evaluation, 4, 307-347.

Savage, R. D. (1984). Alphabet Coding Task-15. Perth, Western Australia: Murdock University.

Tucker-Drob, E. M. (2011). Global and domain-specific changes in cognition throughout adulthood. Developmental Psychology, 47, 331-343.

Wechsler, D. (1986). The Wechsler Adult Intelligence Test - Revised. New York, NY.: The Psychological Corporation.

Wilson, R. S., Beckett, L. A., Barnes, L. L., Schneider, J. A., Bach, J., Evans, D. A., et al. (2002). Individual differences in rates of change in cognitive abilities of older abilities. Psychology and Aging, 17, 179-193. 